
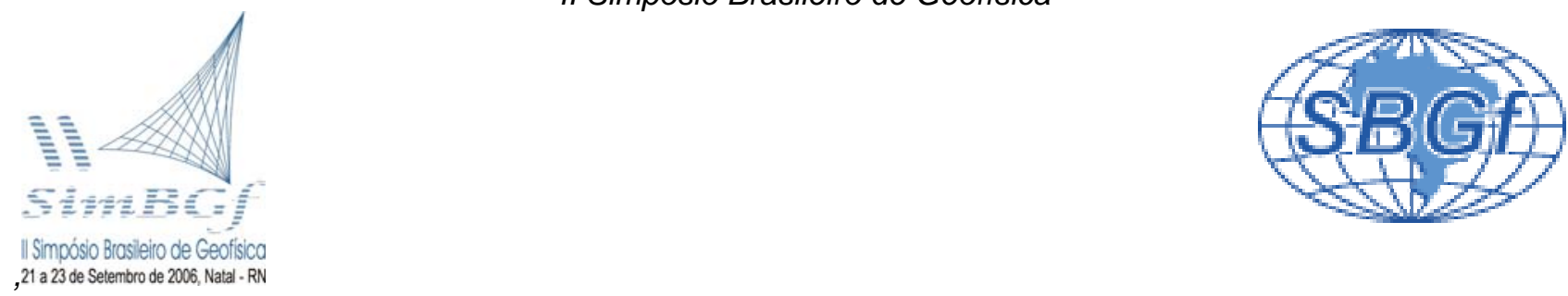

\title{
Imageamento GPR 3-D dos Depósitos Sedimentares da Restinga da Marambaia; Resultados Preliminares
}

Maria C. Pessoa (UFOP), Jandyr M. Travassos (Observatório Nacional)

Email: mariapessoa05@yahoo.com.br; jandyr@on.br

Copyright 2004, SBGf - Sociedade Brasileira de Geofísica

Este texto foi preparado para a apresentação no I Simpósio de Geofísica da Sociedade Brasileira de Geofísica, São Paulo, 26-28 de setembro de 2004. Seu conteúdo foi revisado pela Comissão Tecno-científica do I SR-SBGf mas não neceúciamente representa a opinião da SBGf ou de seus associados. E proibida a necessariamente representa a opinião da SBGf material para propósitos comerciais sem prévia autorização da SBGf.

\section{Resumo}

O presente trabalho apresenta alguns resultados preliminares oriundos de um levantamento de GPR 3-D realizado em depósitos sedimentares do Quaternário que constituem a Restinga de Marambaia. As amostragens espaciais inline e cross-line são iguais, não havendo interpolação de perfis. As antenas foram mantidas na configuração fixed-offset, mantendo as antenas paralelas entre si e perpendiculares aos perfis GPR nas duas direções de medida, evitando-se assim a mistura de polarizações.

Palavras-chave - GPR; 3-D, estratigrafia; alias; sedimentos do Quaternário.

\section{Introdução}

O método GPR é uma técnica de Imageamento eletromagnético reconhecidamente efetiva no estudo da estratigrafia de sedimentos (Davis \& Annan 1989, Gawthorpe et al 1993, McMechan et al. 1997, Van Overmeeren 1998, Vandenberghe \& Van Overmereen 1999, Van Dam \& Schlager, 2000). Estudos de estratigrafia mostram que o GPR, devido a sua resolução e manutenção da continuidade dos refletores em sedimentos, apresenta um potencial até maior que o da sísmica, respeitando o seu limite de alcance em profundidade, (Jol 1995, Mitchum et al. 1977a,b).

Um problema comum em levantamentos GPR 3-D é a ocorrência de alias espacial na direção cross-line, i.e., perpendicular à direção dos perfis. Geralmente a amostragem espacial é correta ao longo dos perfis (direção inline) e completamente inadequada na direção cross-line. Tipicamente a amostragem espacial cross-line é $\geq 4 x$ a amostragem inline. A amostragem não-uniforme força a interpolação de perfis, o que não soluciona, antes agrava ao adicionar outros artefatos, o problema do alias espacial na direção cross-line.

Há na literatura exemplos de levantamentos 3-D com amostragem espacial correta, nas direções inline e crossline (Grasmueck, 1996; Szerbiak et al., 2001; Birken et al., 2002). A literatura também provê instruções para a realização de levantamentos 3-D com amostragem correta nas duas direções, inline e cross-line (Grasmueck \& Weger, 2003).

Note-se aqui que estamos nos concentrando em levantamentos 3-D realizados com as antenas em configuração broadside perpendicular (BP). Esse tipo de levantamento não permite um processamento francamente 3-D, pela introdução forçada de uma polarização distinta na direção cross-line. No presente trabalho a polarização nas duas direções de medida é mantida como BP, evitando-se assim mistura de polarizações. Um exemplo das modificações introduzidas pela mudança de polarização, na região de estudo pode ser encontrada na literatura (Travassos \& André, 2005).

Este trabalho descreve uma etapa preliminar do processamento de um levantamento GPR 3-D realizado na Restinga de Marambaia, RJ, onde as amostragens espaciais inline e cross-line são iguais e a polarização é mantida a mesma nas duas direções. O levantamento foi realizado com configuração fixed-offset, mantendo as antenas paralelas entre si e perpendiculares aos perfis GPR.

\section{A Área de Estudo}

A Restinga de Marambaia, Figura 1, é um patrimônio histórico e ambiental do Estado do Rio de Janeiro, cujo acesso é controlado pelas Forças Armadas. O primeiro trecho da Restinga é utilizado como campo de provas e de treinamento do Exército, ao centro a Aeronáutica e a oeste a Marinha.

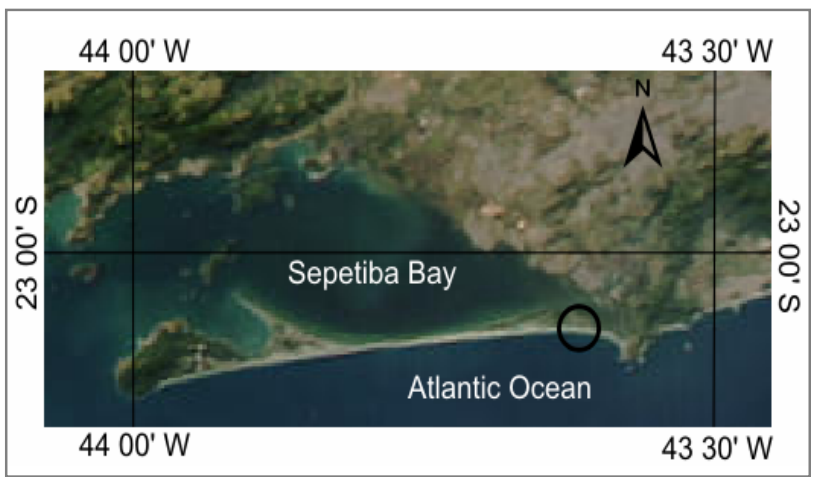

Figura 1. Foto satélite da Restinga de Marambaia. Área de estudo está indicada com circulo preto.

A Restinga apresenta $40 \mathrm{~km}$ de extensão, terminando a oeste, na Ilha de Marambaia. São encontradas diversas 
feições sedimentares atuais e pretéritas, em toda a sua extensão. Identificaram-se dois campos de dunas, a oeste e a norte da área alagada, com dunas de granulometria média, formato arredondado e altura média de 3.0 metros e foram classificadas de parabólicas por Ponçano (1976) e barcanas por Roncati \& Barrocas (1978), estando fixadas por vegetação e sofrem retrabalhamento pelo vento sudoeste, comprovado pela orientação SW-NE dos blow-outs. O processo de sedimentação Quaternária das dunas litorâneas iniciouse no Pleistoceno, durante o período glacial (Borges 1990).

Estudos sobre a evolução geológica da área, em essecial sobre a gênese da Restinga da Marambaia, apresentam controvérsias em relação à fonte de sedimentos durante a formação da Restinga, direção de crescimento e seqüência de aparecimento das diversas feições geomorfológicas (Ponçano et al. 1979).

A coluna sedimentar do campo de dunas está constituída por sedimentos fluviais de canais de maré, mangue e marinhos (Ponçano 1976). Estes sedimentos compõem uma seqüência transgressiva, onde a base está caracterizada por sedimentos de ambiente continental, sobrepostos por sedimentos de ambiente misto que correspondem à subida do nível do mar durante o estágio interglacial. Estes sedimentos foram entalhados durante o período glacial, quando o nível do mar estava a 80 metros abaixo do seu nível atual, tendo então ocorrido à formação de vales atualmente submersos (Borges 1990).

O detalhamento dos depósitos terciários e quaternários que ocorrem ao longo do litoral do Brasil é de fundamental importância para a caracterização dos eventos geológicos que marcaram o período Neógeno na escala mundial, uma vez que eles não se constituem em eventos isolados, mas documentam uma história de flutuação do nível do mar consistente com muitas outras áreas da América do Sul e do mundo, refletindo mudanças eustáticas, provavelmente combinadas a fatores tectônicos (Rossetti, 2001; Rossetti \& Góes,2001)

\section{O Conjunto de Dados}

Foram realizadas 5 campanhas de campo na Restinga da Marambaia, no período de Agosto a Dezembro de 2005. Foram obtidos dados GPR e de topografia sobre uma área de $30 \times 20$ metros. A topografia foi obtida com equipamento DGPS Leica e um nível óptico numa malha de 31 pontos, aproximadamente regular de $5 \times 5$ metros. A topografia foi referenciada a um ponto do IBGE, distante cerca de 500 metros da área de estudo (Figura 2).

Os dados GPR foram obtidos com equipamento Sensor \& Software PE100, com antenas de $100 \mathrm{MHz}$, com janela de tempo de até 850 ns e pulser de $1000 \mathrm{~V}$ ao nível da transmissora. Essa janela de tempo permite uma penetração do sinal até a profundidade da ordem de 50 metros. A distância entre as antenas receptora e transmissora foi mantida fixa a 1 metro. O disparo do GPR foi realizado no tempo e com disparo de linha. Todos os perfis têm 20 metros de comprimento. Neste trabalho concentrar-nos-emos em dois perfis perpendiculares $A$ e $B$, mostrados na Figura 2. Para efeito de apresentação dos resultados restringiremos a janela de tempo a 400 ns.

O processamento dos dados compreendeu uma extensa etapa de edição, seguido de uma etapa de processamento básico, compreendendo dewow, filtragem temporal e espacial e ganho.

\section{Resultados}

As seções de GPR adquiridas apresentaram o padrão de qualidade que permite a interpretação da dinâmica costeira da Restinga de Marambaia, revelando estruturas de dunas, progradações e fácies que caracterizam mudanças do nível do mar.

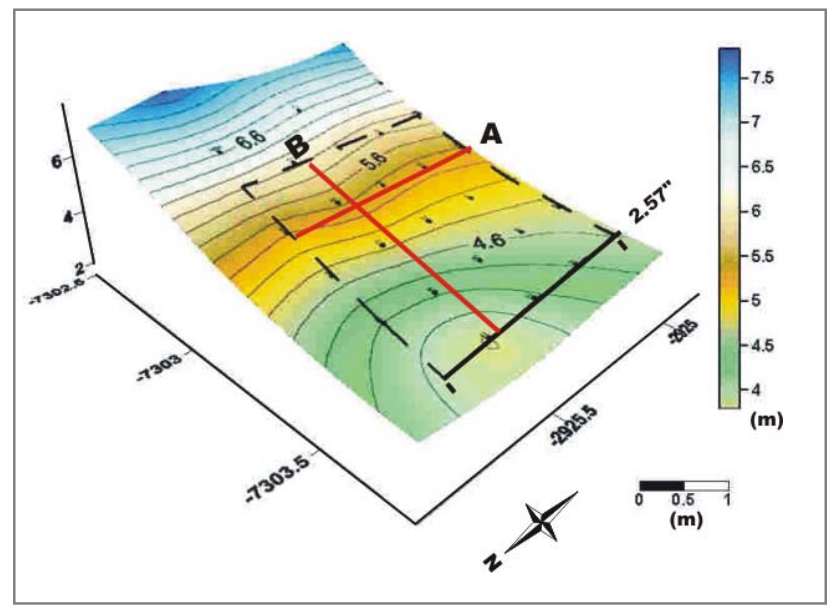

Figura 2 - Localização de dois perfis GPR (A e B) sobre a topografia da área de estudo. Os perfis correm de $\mathbf{N}$ vara $\mathrm{S}$ e $\mathrm{W}$ bara $\mathrm{E}$. respectivamente.

O modelo de velocidade encontrado para esta área é composto possui duas velocidades: 0 - (180 ns) - 0.14 $\mathrm{m} / \mathrm{ns} ; 180 \mathrm{~ns}-0.09 \mathrm{~m} / \mathrm{ns}$. Deste modo podemos estimar que a profundidade de investigação da seção até 645 ns é de 35 metros. A Figura 3 mostra o modelo de velocidade na área.

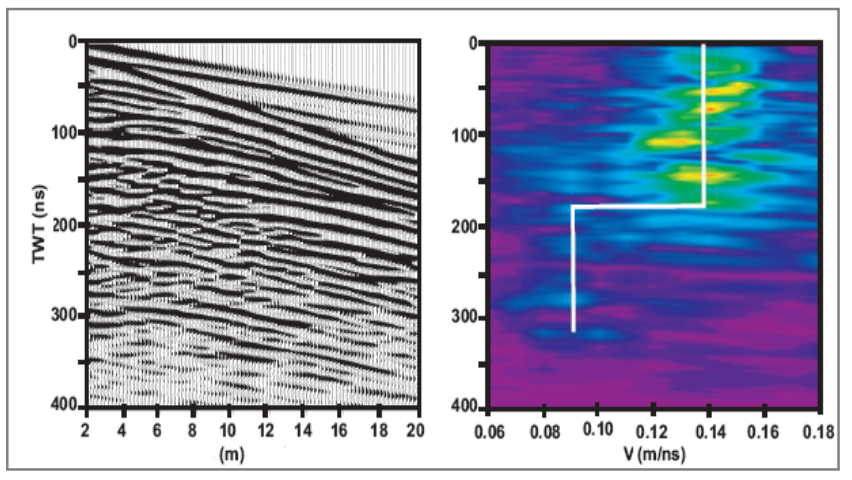

Figura 3 - O painel da esquerda mostra o resultado de um perfil CMP. O painel da direita apresenta o modelo de velocidades. O perfil CMP é colinear com o perfil (A) mostrado na Figura 2. 


\section{Discussão}

A Figura 4 mostra a seção GPR do perfil E-W. A qualidade dos dados é evidente, mostrando todo o potencial do método em reproduzir a estratigrafia da área.

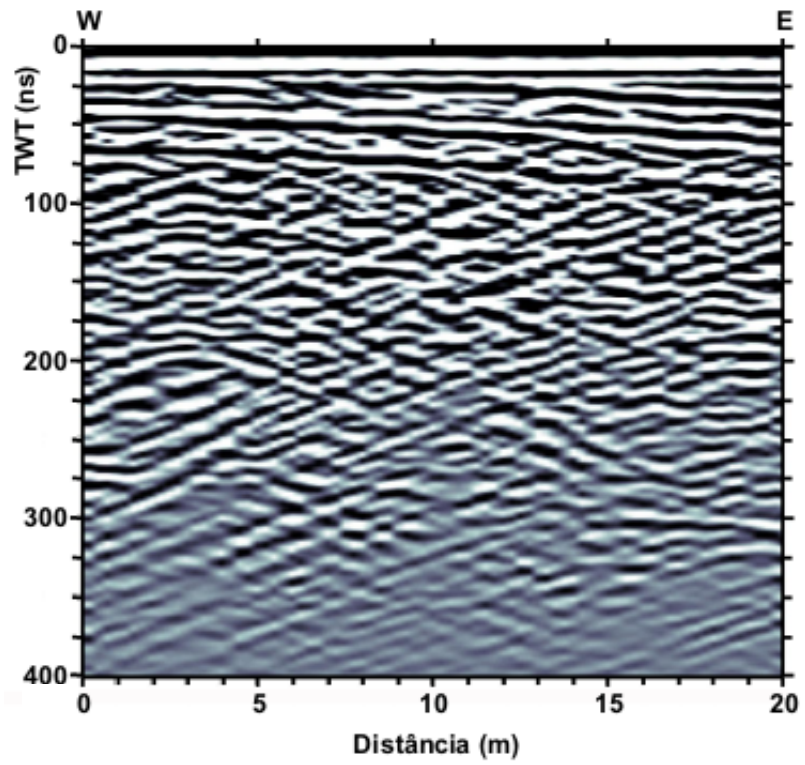

Figura 4 - Seção E-W obtida com configuração fixed offset BP.

A Figura 5 apresenta um diagrama de cerca para os perfis $A$ e $B$, com uma visão desde SE. A continuidade dos refletores nas duas seções é manifesta.

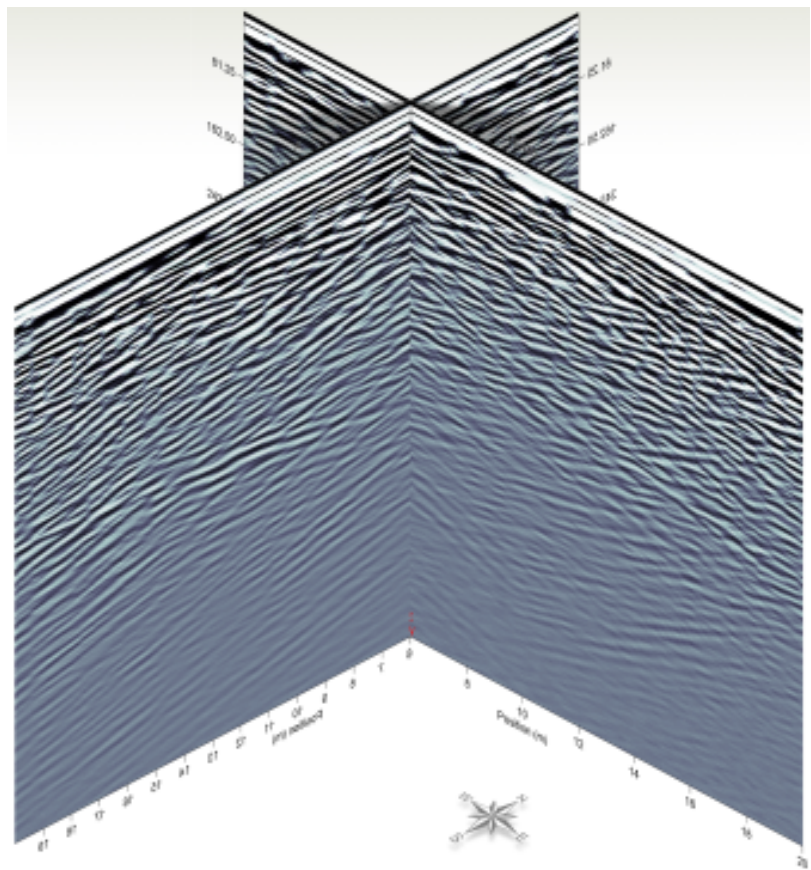

Figura 5 -Diagrama de cerca para os perfis $A$ e $B$, mostrados na Figura 2.

\section{Conclusão}

Este trabalho apresentou alguns resultados preliminares oriundos de um levantamento GPR francamente 3-D, realizado na Restinga de Marambaia. As amostragens espaciais inline e cross-line são iguais, não havendo interpolação de perfis. Manteve-se a mesma polarização das antenas evitando-se assim a mistura de polarizações nas duas direções de medida.

A qualidade dos dados é muito boa, permitindo reproduzir a estratigrafia da área. Este nível de detalhamento permitirá fornecer subsídios ao estudo dos depósitos terciários e quaternários que ocorrem ao longo do litoral do Brasil, os quais por não se constituírem em eventos isolados, documentam uma história de flutuação do nível do mar, consistente com muitas outras áreas da América do Sul e do mundo. A estratigrafia detalhada dos depósitos sedimentares da Marambaia reflete as mudanças eustáticas, provavelmente combinadas a fatores tectônicos, eventos geológicos que marcaram o período Neógeno em escala mundial.

\section{Agradecimentos}

Os recursos para a realização do trabalho de campo foram fornecidos pela PETROBRAS. JT agradece a outorga de Bolsa de Pesquisa do CNPq. Os autores agradecem ao Exército Brasileiro a permissão de utilizar a área da Restinga de Marambaia.

\section{Referências}

Annan, A.P. \& Cosway, S.W., 1992, Ground Penetrating Radar Survey, Design, Annual Meeting of SAGEEP, Chicago, Apnl, 26-29.

Birken, R., Miller, D.E., Burns, M., Albats, P., Casadonte, R., Deming, R., Derubeis, T., Hansen, T., and Oristaglio, M., 2002, efficient large-scale underground utility mapping in New York City using a multi-channel ground-penetrating imaging radar system: Proceedings of SPIE 4758: 186-191.

Borges, H.V.1990, Dinâmica sedimentar da Restinga de Marambaia e Baía de Sepetiba. Dissertação de Mestrado, Departamento de Geologia, Universidade Federal do Rio de Janeiro, pp.

Davis L.J. and Annan A.P. 1989. Ground-penetrating radar for high-resolution mapping of soil and rock stratigraphy. Geophysical Prospecting 37, 531-551.

Jol H.M. 1995. Ground penetrating radar antennae frequencies and transmitter powers compared for penetration depth, resolution and reflection continuity. Geophysical Prospecting 43, 693-709.

McMechan, G. A, Gaynor, G C, and Szerbiak, RB, 1997. Use of ground- penetrating radar for 3-D sedimentological characterization of clastic reservoir analogs. Geophysics, 62, 786-796.

Gawthorpe R.L., Collier R.E.L., Alexander J., Bridge J.S. and Leeder M.R. 1993. Ground penetrating radar: application to sand body geometry and heterogeneity studies. In: Characterization of Fluvial 
and Aeolian Reservoirs (Ends C.P. North and D.J. Prosser), Special Publication 73, pp. 421-432. The Geological Society, London.

Grasmueck, M., 1996, 3-D Ground-penetrating radar applied to fracture imaging in gneiss: Geophysics, 61: 1050-1064.

Grasmueck, M., Weger, R., 2003, How dense is dense enough for a 'real' 3D GPR Survey, SEG 2003, Dallas: $4 p$.

McMechan, G. A, Gaynor, G C, \& Szerbiak, RB, 1997. Use of ground- penetrating radar for 3-D sedimentological characterization of clastic reservoir analogs. Geophysics, 62, 786-796.

Mitchum R.M., Vail P.A \& Thompson S. III 1977a. Seismic stratigraphy and global changes of sea level; part 2: The depositional sequence as a basic unit for seismic stratigraphic analysis. In: Seismic Stratigraphy - Application to Hydrocarbon Exploration (ed.C.E. Payton), Memoirs 26, pp. 53-62.American Association of Petroleum Geologists.

Mitchum R.M., Vail P. A \& Sangree J.B. 1977b. Seismic stratigraphy and global changes of sea level; part 6: Stratigraphic interpretation of seismic reflection patters in depositional sequences. In: Seismic Stratigraphy - Application to Hydrocarbon Exploration (ed. C.E. Payton), Memoirs 26, pp. 117133.American Association of Petroleum Geologists.

Ponçano, W.L.1976. Sedimentação atual na Baía de Sepetiba, Estado do Rio de Janeiro: um estudo para a avaliação da viabilidade geotécnica de implantação de um porto. Dissertação de Mestrado; Instituto de Geociências, Universidade de São Paulo, São Paulo, pp.

Ponçano, W.L., Fulfaro, V.J. \& Gimenez, A.F., 1979. Sobre a origem da Restinga de Marambaia, RJ.In: Simpósio Regional de Geologia, 2, Rio Claro. Atas...Rio Claro, SP, v.1,p.291-304

Roncarati, H. \& Barrocas, S.L.S., 1978. Projeto Sepetiba. Estudo geológico preliminar dos sedimentos recentes superficiais da Baía de Sepetiba-município do Rio de Janeiro, Itaguaí e Mangaratiba - RJ, PETROBRAS. CENPES (Relatório preliminar), 35p.

Szerbiak, R.B., McMechan, G.A., Corbeanu, R., Forster, C.B.; Snelgrove, S.H., 2001, 3-D characterization of a clastic reservoir analog; from 3-D GPR data to a 3-D fluid permeability model: Geophysics, 66: 1026-1037.

Travassos, J.M., André, S.S., 2005, Polarization issues in a recent sedimentation Environment, 9th International Congress of the Brazilian Geophysical Society, Salvador, 11-14 de Set., Extended Abstract (117) in CD ROM.

Van Overmeeren R.A. 1998. Radar facies of unconsolidated sediments in The Netherlands: A radar stratigraphy interpretation method for hydrogeology. Journal of Applied Geophysics 40, $1-18$.
Vandenberghe J. \& Van Overmereen R.A. 1999. Ground penetrating radar images of selected fluvial deposits in the Netherlands. Sedimentary Geology 128, 245270.

Van Dam R.L \& Schlager W. 2000. Identifying causes of ground penetrating Radar reflections using timedomain reflectometry and sedimentological Analyses Sedimentology 47, 435-449. 